\title{
(2) OPEN ACCESS \\ Intraoperative frozen section consultation by remote whole-slide imaging analysis -validation and comparison to robotic remote microscopy
}

\author{
Thomas Menter (ㄷ), Stefan Nicolet, Daniel Baumhoer, Markus Tolnay, \\ Alexandar Tzankov
}

Institute of Pathology and Medical Genetics, University Hospital Basel, Basel, Switzerland

\section{Correspondence to} Professor Alexandar Tzankov, Pathology, University Hospital Basel, Basel 4031, Switzerland; alexandar.tzankov@usb.ch

SGPath Annual Meeting November 7-9 2019, Lucerne, Switzerland

Received 10 October 2019 Revised 1 November 2019 Accepted 1 November 2019 Published Online First 12 November 2019
Check for updates

(C) Author(s) (or their employer(s)) 2020. Re-use permitted under CC BY-NC. No commercial re-use. See rights and permissions. Published by BMJ.

To cite: Menter T,

Nicolet S, Baumhoer D,

et al. J Clin Pathol

2020;73:350-352

\begin{abstract}
Digital pathology including whole slide image (WSI) acquisition is a promising tool for histopathologic teleconsultation. To test and validate the use of WSI in comparison with robotic microscopy for intraoperative frozen section consultation of peripheral hospitals serviced by our department, we compared the VENTANA DP 200 slide scanner with an established remote-controlled digital microscope. Thirty cases were retrospectively analysed. In comparison with a median specimen handling time of 19 min using remote-controlled microscopy, the WSI handling was significantly shorter ( $11 \mathrm{~min}, \mathrm{p}=0.0089)$ and offered better image quality, for example, allowing to detect a positive resection margin by a malignant melanoma that had been missed using the former system. Prospectively assessed on 12 cases, the median handling time was $6 \mathrm{~min}$. Here, we demonstrate the applicability and the advantages of WSI for intraoperative frozen section teleconsultation. WSI-based telepathology prooves to be an efficient and reliable tool providing superior turnaround time and image resolution.
\end{abstract}

\section{INTRODUCTION}

Since the introduction of telepathology almost 30 years ago, ${ }^{1}$ much progress has been made in digitalisation of pathology. ${ }^{2}$ A major break-through is the widespread availability of whole slide imaging (WSI) that provides image quality identical to conventional light microscopes next to many other advantages. ${ }^{3}$ Moreover, deep learning approaches and automated image analysis are about to be integrated into surgical pathology routine practice in the near future. ${ }^{45}$

Telepathology, both in real time, for example, in the setting of frozen section intraoperative consultation, and time-shifted, is becoming a more and more important tool as it helps to provide pathology services in hospitals lacking a pathology department and can be used as a consultation tool for expert opinions. ${ }^{6}$ Telepathology overcomes the need to physically send slides and blocks to other departments and thus improves the time needed to reach a definitive diagnosis and the availability of expert knowledge. ${ }^{7}$

In the present study, we describe our experience in using a slide scanner and its affiliated image management software (IMS) in the setting of intraoperative frozen section consultation for the Hôpital du Jura in Delémont, Switzerland, located
$40 \mathrm{~km}$ away from our institute, which is located in Basel, Switzerland. We also compare our findings with our previously established solution in the form of a remote-controlled digital microscopy. ${ }^{8}$

\section{MATERIALS AND METHODS}

We retrospectively reanalysed 30 consecutive cases of intraoperative frozen section consultations which have already been worked-up by remote-controlled microscopy to validate the new WSI system. The initial diagnoses were blinded and compared with the diagnoses achieved by WSI subsequently. The cases consisted of 8 axillary sentinel lymph nodes of breast cancers, 14 skin excisions for basal cell or squamous cell carcinoma or melanoma, 4 cases of resection margins of gastrectomies, 1 ovarian and 1 renal tumour, 1 focal peritoneal fatty tissue necrosis, and 1 clinically suspicious gall bladder. Each slide contained two serial sections of the specimen. Initially, the cases have been analysed at the time of actual surgery using our previous system iPath (www.ipath-network.org), a web-based teleconsultation system for surgical pathology. ${ }^{8}$ In this setup, the frozen section was evaluated using a Nikon COOLSCOPE microscope (Nikon GmbH, Egg, Switzerland) that was remote-controlled by a pathologist via iPath. This system has been in use since 2001 and for the purposes of intraoperative frozen section consultation in the Hôpital du Jura since 2008.

In the new setting, the slides were scanned using a VENTANA DP 200 slide scanner (Roche Diagnostics, Rotkreuz, Switzerland) and its affiliated image management software (IMS) uPath. Due to time restraints in the setting of intraoperative frozen section consultation, we limited scanning to a single focus layer. The technician adjusted the regions of interest to two complete serial sections of the specimen per slide to warrant comparability with the previously used methodology. Thus, small foci of non-analysable tissue fragments included in the scanning area by the computer were omitted. Digital pathology, in general, was established at our institute in 2018 and validated by scanning 500 routine formalin-fixed paraffin-embedded cases (according to the guidelines of the College of American Pathologists). ${ }^{9}$ All cases were viewed independently by two pathologists (TM and AT) using both WSI and glass slides.

We also verified our findings on 12 prospectively analysed cases (6 axillary sentinel lymph nodes of 
Table 1 Details of the cases investigated as well as the technical procedures

\begin{tabular}{|c|c|c|c|}
\hline & Median & Mean & Range \\
\hline Slides per case & 2 & 2.5 & $1-5$ \\
\hline Time for scanning* $(\min )$ & 4 & 3 & $1-9$ \\
\hline Time for transmission to uPath* (min) & 3 & 3 & $2-7$ \\
\hline Handling time of the pathologist* (min) & 2 & 2 & $1-7$ \\
\hline Total handling time of the technician* (min) & 9 & 9 & $5-18$ \\
\hline Total time for WSI approach* (min) & 11 & 11 & $6-22$ \\
\hline $\begin{array}{l}\text { Total handling time of the pathologist using } \\
\text { robotic telemicroscopy (min) }\end{array}$ & 16 & 21 & $6-61$ \\
\hline $\begin{array}{l}\text { Total handling time of the pathologists using WSI } \\
\text { and Ventana Image Viewer }\end{array}$ & 6 & 11 & $4-46$ \\
\hline
\end{tabular}

*These are times per case and not per slide as the cases were scanned batchwise simulating the procedures during real-time frozen section analysis. As transferring of the files to uPath started already while the later slides were still in the scanning process, scanning and data transfer of single slides cannot be given for all cases. WSI, whole slide imaging.

breast cancer, 4 skin excisions, 1 cervical lymph node and 1 submandibular gland) in the first 6 months of 2019, which have also been scanned by the DP 200 slide scanner, yet the slides could not be transferred to uPath as the link to our laboratory information system was not yet established. Instead, the slides were transferred by the technician in Delémont to a virtual drive and analysed by the pathologist using the Ventana Image Viewer v.3.2.0 (Ventana Medical Systems, Tucson, Arizona, USA).

The image quality was assessed in a six-tier system ranging from 'very good/the same as a slide under a conventional microscope' (1) to 'not suitable for diagnostics' (6). If the slide had to be rescanned or was partially out of focus, the final grade was put one tier less than the actual image quality in order to address also the required speed of the availability of diagnostic material.

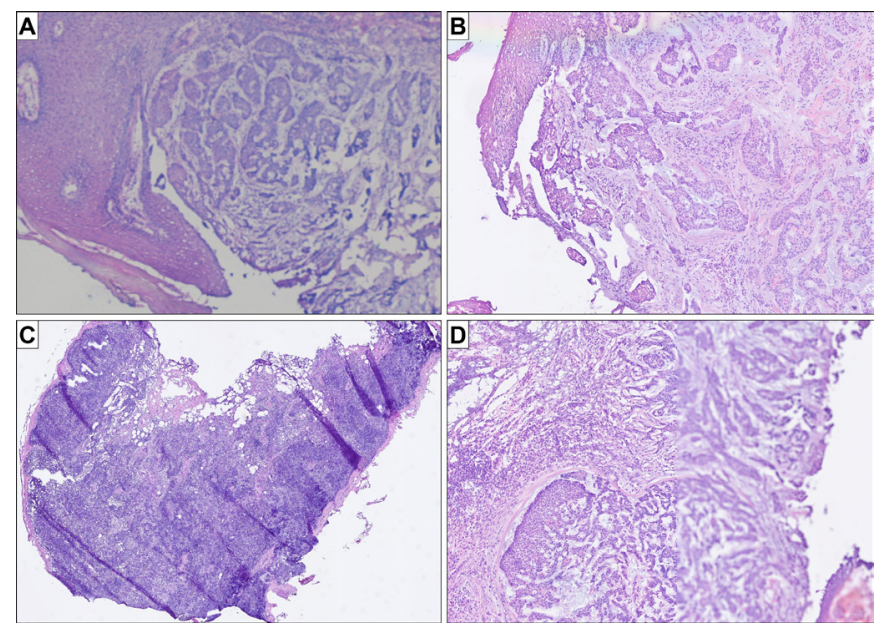

Figure 2 Histology images and potential pitfalls and advantages of WSI (all figures represent original images without postprocessing). (A) routine quality of $\mathrm{H} \& \mathrm{E}$ frozen sections slides using robotic microscopy and the iPath system (basal cell carcinoma); (B) routine quality of the same H\&E frozen section slide using uPath; (C) case with many folds, in which the IMS managed to correctly focus on the thin evaluable areas without the need of further manual readjustment (sentinel lymph node); (D) case with a focus issue in one of the serial sections: while the left half of the tissue is focused correctly, the right half is out of focus; the second serial section was entirely focused (basal cell carcinoma of $B$ ). IMS, image management software; WSI, whole slide imaging.
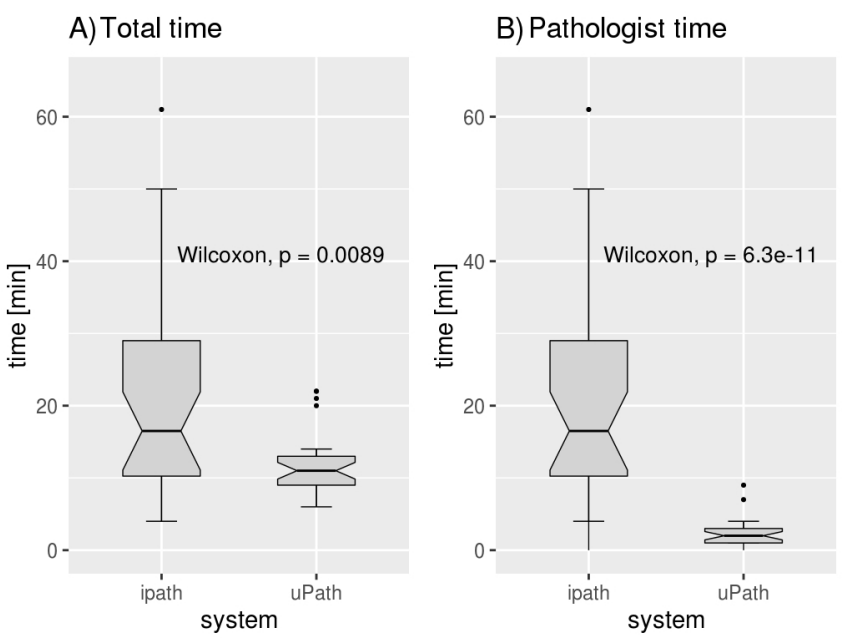

Figure 1 Box plots comparing the total time from having the slide ready for analysis to making the diagnosis in intraoperative frozen section consultation: total time using WSI (left), time needed by the pathologist using WSI (middle) and time need by the pathologist using iPath (right). WSI, whole slide imaging.

Times for macroscopy and slide preparation at the peripheral site were not assessed as they would not have varied between the two settings of WSI and robotic microscopy.

Statistics and graphics were done with the software R V.3.6.1 using the ggpubr package V.0.2.2, available at https:/github.com/ kassambara/ggpubr. The paired Wilcoxon test was as the normal distribution does not apply to both the total time of pathologist and for using uPath.

\section{RESULTS}

All cases could be successfully analysed using the VENTANA DP 200 slide scanner. The median handling time of the technicians' work for the scanning procedure and the transfer to uPath was $9 \mathrm{~min}$. The median time required by the pathologist for each case using uPath to reach a diagnosis was $2 \mathrm{~min}$. The median total handling time from the slide being ready for scanning to the diagnosis transmitted to the surgeon by the pathologist was 11 min (table 1, figure 1).

One case had to be scanned twice as it was out of focus, and in 3 other cases, one of the serial sections was out of focus, but the diagnosis could still be made using the remaining serial section. The main reasons for focus issues were tissue folds, a common problem in fresh frozen sections as the tissue is often not homogeneous and the sections are, for physical reasons, relatively thick.

All WSI-based diagnoses were identical to the final diagnosis made after paraffin embedment and conventional microscopy. In contrast, a positive resection margin of a melanoma in situ which had been initially missed using remote-controlled microscopy was identified using WSI.

Compared with the established system using a remotecontrolled microscope, the handling time by the pathologist using WSI was significantly reduced (median time of 2 vs $16 \mathrm{~min}$, respectively, $\mathrm{p}=0.0089$ ) as the slides could be evaluated much quicker using uPath. Image quality and resolution were also significantly improved using WSI (median value by WSI: 1 (range: 1-3), median value by robotic microscopy: 3 (range: $2-4), p<0.001)$. On the other hand, robotic microscopy allowed the user to re-focus the slide at any time, so focus issues were not a problem using that technique (figure 2). 
In our prospective hands-on practical approach using the Ventana Image Viewer to evaluate scanned slides, the median time per case required by the pathologist was 6 min (range: 4-46 min). In three cases, the pathologist had to work on individual cases $>10$ min due to technical problems such as images with focus issues or considerable diagnostic challenges (three large sentinel lymph nodes, meticulous analysis of the resection margins in a case of melanoma in situ).

\section{DISCUSSION}

Here we present a feasible approach for intraoperative frozen section consultation using WSI. This approach has an equivalent image quality compared with on-site evaluation using a conventional microscope. We measured a median diagnosis time of eleven minutes to scan the slide, transfer the data and establish the diagnosis. Compared with other telepathology studies on intraoperative frozen section consultation using different types of telepathology, we achieved an improved turnaround time and image quality using WSI. ${ }^{10}$ We furthermore compared two different softwares for analysing the images with uPath having the advantage of being an integrative software both for laboratory management (possibility of a direct and documented assignment of slides to a case and to the respective pathologist) as the Ventana Image viewer has just been designed to analyse image files.

We also observed that WSI of frozen tissue sections with an average thickness of $8-12 \mu \mathrm{m}$ is more challenging than that of average paraffin sections of $2-3 \mu \mathrm{m}$ thickness, as the focus point can be lost during the scanning process, especially if the sections contain many folds. We needed to re-scan one slide completely, and, in three other cases, one of the serial sections was partially out of focus, which nonetheless did not impede reaching the correct diagnosis (infiltration of basal cell carcinoma or squamous cell carcinoma in the resection margins). As the focus point can be adjusted manually, it is important to control and, if necessary, set it before the start of the scanning process.

Although not the focus of this study, it is important to state that establishing a WSI scanner together with its IMS faces many information technology challenges and requires a robust local network and servers as well as extensive testing at both sites. Furthermore, we comprehensively trained our laboratory and informatics technicians and involved pathologists to enable reliable use of the scanner and the software. Technicians must know how to prepare and focus the tissue slides to prevent delaying the transmission of the diagnosis. Sections should be cut as thin as possible and ideally should not contain folds that can impede the focus procedures of the instrument. Pathologists must be aware of these technical artefacts and be ready to communicate problems with the laboratory technicians at the remote site.

To conclude and in agreement with other groups ${ }^{11} 12$ using other scanning and software platforms, we demonstrate the feasibility of WSI for telepathology in the context of intraoperative frozen section consultation. We compared our results with our long experience in remote-controlled microscopy and showed considerable improvement in the image quality and handling time. The success of telepathology with WSI depends on a comprehensive integration of the slide scanning process into the routine workflow, high usability and availability of the image management system, a stringent IT support and experienced and dedicated technicians and pathologists.

Handling editor Runjan Chetty.

Acknowledgements The authors would like to thank Roche Diagnositics for providing access to a DP 200 scanner and the uPath software.

Contributors TM, DB, MT and AT: designed the study. TM and SN: performed the accrual of the data of the retrospective analysis. TM and AT: accrued the data of the iPath cases and the prospective cases using the Ventana Image Viewer. TM: wrote the manuscript. All authors read and approved the manuscript.

Funding The authors have not declared a specific grant for this research from any funding agency in the public, commercial or not-for-profit sectors.

Competing interests None declared.

Patient consent for publication Not required.

Provenance and peer review Not commissioned; internally peer reviewed.

Open access This is an open access article distributed in accordance with the Creative Commons Attribution Non Commercial (CC BY-NC 4.0) license, which permits others to distribute, remix, adapt, build upon this work non-commercially, and license their derivative works on different terms, provided the original work is properly cited, appropriate credit is given, any changes made indicated, and the use is non-commercial. See: http://creativecommons.org/licenses/by-nc/4.0/.

ORCID iD

Thomas Menter http://orcid.org/0000-0002-0847-6156

\section{REFERENCES}

1 Nordrum I, Engum B, Rinde E, et al. Remote frozen section service: a telepathology project in northern Norway. Hum Pathol 1991;22:514-8.

2 Griffin J, Treanor D. Digital pathology in clinical use: where are we now and what is holding us back? Histopathology 2017;70:134-45.

3 Zarella MD, Bowman; D, Aeffner F, et al. A practical guide to whole slide imaging: a white paper from the digital pathology association. Arch Pathol Lab Med 2019;143:222-34.

4 Schaer R, Otálora S, Jimenez-del-Toro 0, et al. Deep learning-based retrieval system for gigapixel histopathology cases and the open access literature. J Pathol Inform 2019;10:19.

5 Wang S, Yang DM, Rong R, et al. Pathology image analysis using segmentation deep learning algorithms. Am J Pathol 2019.

6 Brauchli K, Oberli H, Hurwitz N, et al. Diagnostic telepathology: long-term experience of a single institution. Virchows Archiv 2004;444:403-9.

7 Farahani N, Pantanowitz L. Overview of Telepathology. Surg Pathol Clin 2015;8:223-31.

8 Brauchli K, Christen H, Haroske G, et al. Telemicroscopy by the Internet revisited. J Pathol 2002;196:238-43.

9 Pantanowitz L, Sinard JH, Henricks WH, et al. Validating whole slide imaging for diagnostic purposes in pathology: guideline from the College of American pathologists pathology and laboratory quality center. Arch Pathol Lab Med 2013; 137:1710-22.

10 Evans AJ, Chetty R, Clarke BA, et al. Primary frozen section diagnosis by robotic microscopy and virtual slide telepathology: the University health network experience. Hum Pathol 2009;40:1070-81.

11 Cima L, Brunelli M, Parwani A, et al. Validation of remote digital frozen sections for cancer and transplant intraoperative services. J Pathol Inform 2018;9.

12 French JMR, Betney DT, Abah U, et al. Digital pathology is a practical alternative to on-site intraoperative frozen section diagnosis in thoracic surgery. Histopathology 2019;74:902-7. 